\title{
Two faces of quantum sound
}

\author{
C. Barceló, ${ }^{1}$ L. J. Garay, ${ }^{2,3,4}$ and G. Jannes ${ }^{1,3,5}$ \\ ${ }^{1}$ Instituto de Astrofísica de Andalucía (IAA-CSIC), Glorieta de la Astronomía, 18008 Granada, Spain \\ ${ }^{2}$ Departamento de Física Teórica II, Universidad Complutense de Madrid, 28040 Madrid, Spain \\ ${ }^{3}$ Instituto de Estructura de la Materia (IEM-CSIC), Serrano 121, 28006 Madrid, Spain \\ ${ }^{4}$ King's College London, Department of Physics, Strand, London WC2R 2LS, United Kingdom \\ ${ }^{5}$ Université de Nice Sophia Antipolis, Laboratoire J.-A. Dieudonné, UMR CNRS-UNS 6621, Parc Valrose, 06108 Nice Cedex 02, \\ France
}

(Received 2 June 2010; revised manuscript received 2 July 2010; published 25 August 2010)

\begin{abstract}
Fluctuations around a Bose-Einstein condensate can be described by means of Bogolubov theory leading to the notion of quasiparticle and antiquasiparticle familiar to nonrelativistic condensed-matter practitioners. On the other hand, we already know that these perturbations evolve according to a relativistic Klein-Gordon equation in the long-wavelength approximation. For shorter wavelengths, we show that this equation acquires nontrivial corrections which modify the Klein-Gordon product. In this approach, quasiparticles can also be defined (up to the standard ambiguities due to observer dependence). We demonstrate that-in the low-energy as well as in the high-energy regimes-both concepts of quasiparticle are actually the same, regardless of the formalism (Bogolubov or Klein-Gordon) used to describe them. These results also apply to any barotropic, inviscid, irrotational fluid, with or without quantum potential. Finally, we illustrate how the quantization of these systems of quasiparticles proceeds by analyzing a stationary configuration containing an acoustic horizon. We show that there are several possible choices of a regular vacuum state, including a regular generalization of the Boulware vacuum. Issues such us Hawking radiation crucially depend on this vacuum choice.
\end{abstract}

DOI: 10.1103/PhysRevD.82.044042

PACS numbers: 04.62.+v, 03.75.Kk, 04.70.Dy

\section{INTRODUCTION}

It has for quite some time been understood that the propagation of sound waves in inviscid irrotational barotropic fluids can, under rather general circumstances, be effectively described by relativistic curved-spacetime metrics; see e.g. [1]. This led to the observation that it should, at least in principle, be possible to achieve sonic or acoustic black hole configurations, and thus study certain types of high-energy effects on black hole physics by analogy [2]. This gravitational analogy was established on firm foot about a decade ago in [3]. Since then, the field of analogue gravity has grown into a mature and well-established research programme within the gravity community [4]. It offers both the exciting prospect of bringing black hole physics, in particular, Hawking radiation, within the reach of experimentation, and a refreshing conceptual take on some long-standing problems of quantum gravity, such as its relation to dark energy [5] or the avoidance of black hole singularities in the gravitational collapse of ultraheavy bodies [6].

The quest for a useful background fluid in which to conduct experiments of analogue gravity leads in the direction of superfluids. Indeed, superfluids have a vanishing viscosity, and some superfluids can be made extremely pure. Among the possible candidates, Bose-Einstein condensates $[7,8]$ have the comparative advantage of being conceptually well understood and relatively simple to describe theoretically and manipulate experimentally. Acoustic black holes have been recently reported for the first time in Bose-Einstein condensates [9], and there is good hope that an experimental detection of analogue Hawking radiation, one of the main current promises of the analogue gravity programme, will be achievable in the near future [10].

Another motivation for studying Bose-Einstein condensates in the context of analogue gravity is that they provide a real system in which high-energy modifications to the relativistic dispersion relations arise. This shows that there exist concrete examples in nature in which Lorentz invariance is realized as a low-energy effective symmetry, broken at high energy. Such a scenario could be the case for the local Lorentz invariance of general relativity as well. In this manner, Bose-Einstein condensates might offer an interesting model for quantum gravity phenomenology; see e.g. $[11,12]$.

Our first aim in this paper is to further elaborate and consolidate the theoretical framework for the gravitational analogy in Bose-Einstein condensates by taking a look at the different inner products and related creation and annihilation variables that can be introduced depending on the point of view. Quantum sound in Bose-Einstein condensates can, on the one hand, be analyzed within the Bogolubov formalism by directly perturbing the GrossPitaevskii equation. On the other hand, the phase perturbations of the condensate obey a modified Klein-Gordon equation, and a corresponding quantization can be carried out. Remarkably, both procedures give rise to the same quantum theory. This allows us to establish a deep con- 
ceptual connection between both formalisms, the first one being inherently nonrelativistic while the second is relativistic, up to corrections which are vanishingly small for long wavelengths. Both procedures are known to be equivalent in this long-wavelength acoustic limit; see e.g. [13]. Here we discuss in detail how the usual Klein-Gordon field is distorted after the acoustic approximation is broken. The step-by-step analysis that we perform shows that the equivalence between the Bogolubov approach and this generalized Klein-Gordon formalism persists well beyond the limit of validity of the acoustic approximation.

Once an inner product has been defined, one can proceed with the quantization of the system of quasiparticles in the standard Fock manner. An orthonormal and complete set of positive norm modes is needed to expand the field operator. Finding such a complete set of positive norm modes amounts to defining quasiparticle creation and annihilation operators and a vacuum state. This construction is not unique: One can choose several distinct sets of modes and vacuum states which give place to different quasiparticle notions. To illustrate this procedure we will consider a stationary one-dimensional configuration possessing an acoustic black hole horizon. The second aim of this paper is precisely to show that for this configuration there exist several regular vacuum states with a specific interpretation, including a regular generalization of the Boulware state for a relativistic field in a black hole geometry. In this way, we show that the freedom in choosing a vacuum state is larger in dispersive theories than in relativistic theories. This vacuum choice has crucial importance in issues such as the presence or not of Hawking radiation.

This paper is organized as follows. Section II is devoted to the Bogolubov approach and the definition of the appropriate inner product. A mode analysis is also performed. Section III introduces the hydrodynamic representation that leads to a Klein-Gordon equation in the longwavelength regime and to its generalization for all wavelengths. The corresponding generalized Klein-Gordon product is also introduced. In Sec. IV, we show that both formalisms are actually equivalent and lead to the same concept of positive and negative norm solutions. After defining the appropriate inner product, Sec. V is devoted to a discussion of the different vacuum state choices. We focus on a configuration of particular relevance for analogue gravity experiments: a one-dimensional stationary acoustic black hole. We conclude with some final comments in Sec. VI.

\section{BOGOLUBOV APPROACH}

Let us consider a condensed dilute gas of interacting bosons described in terms of quantum field operators $\hat{\psi}$ and $\hat{\psi}^{\dagger}$ that annihilate and create particles (see e.g. Refs. $[14,15])$. The operator $\hat{\psi}$ can be separated into two parts: a macroscopic wave function $\psi_{0}$ describing the actual Bose-Einstein condensate and a quantum field operator $\hat{\phi}$ describing perturbations around the condensate.

The order parameter $\psi_{0}$ satisfies the Gross-Pitaevskii equation

$$
i \hbar \partial_{t} \psi_{0}=\left(-\frac{\hbar^{2}}{2 m} \nabla^{2}+V_{\mathrm{ext}}+g\left|\psi_{0}^{2}\right|\right) \psi_{0},
$$

where $V_{\text {ext }}$ is an external potential, $m$ the atomic mass, and $g$ the atomic interaction constant (proportional to the $s$-wave scattering length). We will also use the Madelung representation

$$
\psi_{0}=\sqrt{n_{0}} e^{i \theta_{0} / \hbar},
$$

in terms of the number density of atoms $n_{0}$ and the phase $\theta_{0}$ of the condensate, which defines the flow velocity potential (such that the flow velocity is $\mathbf{v}=\nabla \theta_{0} / \mathrm{m}$ ). Then Eq. (1) translates into

$$
\begin{gathered}
\partial_{t} n_{0}+\nabla \cdot\left(n_{0} \nabla \theta_{0}\right) / m=0, \\
\partial_{t} \theta_{0}+\frac{1}{2 m}\left(\nabla \theta_{0}\right)^{2}+V_{\text {ext }}+g n_{0}-\frac{\hbar^{2}}{2 m} \frac{\nabla^{2} \sqrt{n_{0}}}{\sqrt{n_{0}}}=0,
\end{gathered}
$$

which are the continuity equation and the Bernoulli equation plus a quantum potential term, respectively.

\section{A. Bogolubov equation}

The quantum perturbation field $\hat{\phi}$ satisfies the Bogolubov equation (see e.g. Refs. $[14,15]$ )

$$
i \hbar \partial_{t} \hat{\boldsymbol{\phi}}=\mathcal{H} \hat{\boldsymbol{\phi}}+m c^{2} e^{2 i \theta_{0} / \hbar} \hat{\boldsymbol{\phi}}^{\dagger},
$$

where $c^{2}=g n_{0} / m$ is the square of the local comoving speed of sound and $\mathcal{H}$ is the operator,

$$
\mathcal{H}=-\frac{\hbar^{2}}{2 m} \nabla^{2}+\frac{\hbar^{2}}{2 m} \frac{\nabla^{2} c}{c}-\frac{1}{2} m \mathbf{v}^{2}-\partial_{t} \theta_{0}+m c^{2} .
$$

The commutation relation for the atomic creation and annihilation operators,

$$
\left[\hat{\psi}(\mathbf{x}, t), \hat{\psi}^{\dagger}\left(\mathbf{x}^{\prime}, t\right)\right]=\delta\left(\mathbf{x}-\mathbf{x}^{\prime}\right),
$$

translates into the commutation relation

$$
\left[\hat{\phi}(\mathbf{x}, t), \hat{\phi}^{\dagger}\left(\mathbf{x}^{\prime}, t\right)\right]=\delta\left(\mathbf{x}-\mathbf{x}^{\prime}\right)
$$

for the perturbation field operators, which create or annihilate atoms in the noncondensed part and correspondingly annihilate or create them in the condensed phase. In other words, $\hat{\phi}$ moves an atom from the noncondensed part to the condensate, and vice versa for $\hat{\phi}^{\dagger}$. This commutation relation is valid for condensed systems in which the number of noncondensed atoms is very small compared to the number of condensed ones.

Note that the Bogolubov equation (5) could also have been obtained by expanding to first order in $\phi$ the Gross- 
Pitaevskii equation (1) for the order parameter $\sqrt{n_{0}} e^{i \theta_{0} / \hbar}+$ $\phi$. In other words, the classical perturbation $\phi$ of the meanfield wave function of the condensate satisfies exactly the same evolution equation as the quantum fluctuations $\hat{\phi}$ around the condensed phase. From now on, we will therefore drop the hat from the operator $\hat{\phi}$, unless necessary.

It is important to note that, although this equation can be obtained by "linearizing" the Gross-Pitaevskii equation, it is a complex equation for a genuinely complex field and is therefore nonlinear: If $\phi$ is a solution, then, in general, $\alpha \phi$ is not (unless $\alpha$ is real). Therefore, we cannot directly perform a mode expansion to find the general solution. There exists a procedure $[14,15]$ which allows us to overcome this problem by enlarging the space in which we look for solutions to Eq. (5) and to define an inner product in this enlarged space.

With this aim let us introduce the spinor field

$$
\Phi=\frac{1}{\sqrt{2}}\left(\begin{array}{c}
\phi \\
\tilde{\phi}
\end{array}\right)
$$

subject to the evolution equation

$$
i \hbar \partial_{t} \Phi=\mathcal{M} \Phi,
$$

where $\mathcal{M}$ is the operator

$$
\begin{aligned}
\mathcal{M} & =\left(\begin{array}{cc}
\mathcal{H} & m c^{2} e^{2 i \theta_{0} / \hbar} \\
-m c^{2} e^{-2 i \theta_{0} / \hbar} & -\mathcal{H}
\end{array}\right) \\
& =\mathcal{H} \sigma_{z}+m c^{2} e^{2 i \theta_{0} / \hbar} \sigma_{+}-m c^{2} e^{-2 i \theta_{0} / \hbar} \sigma_{-} .
\end{aligned}
$$

In this equation, $\sigma_{ \pm}=\left(\sigma_{x} \pm i \sigma_{y}\right) / 2$ and $\sigma_{x, y, z}$ are the Pauli matrices.

This equation is now linear; i.e., if $\Phi$ is a solution, then so is $\alpha \Phi$ for any complex constant $\alpha$. The solutions to the Bogolubov equation (5) are obtained by restricting the solutions of Eq. (10) by the condition

$$
\phi^{*}=\tilde{\phi}, \quad \text { i.e., } \quad \sigma_{x} \Phi^{*}=\Phi .
$$

\section{B. Bogolubov inner product}

Taking into account that $\hat{\phi}$ has been defined as an operator that annihilates atoms in the noncondensed part of the gas (and hence creates them in the condensed part), the expectation value of $\hat{\phi}^{\dagger} \hat{\phi}$ will provide the number of noncondensed atoms $N_{1}$ (under the assumption that this number is small compared to the total number of atoms, as mentioned above). This condition translates into the following normalization condition for the spinor $\Phi$ :

$$
\langle\Phi, \Phi\rangle=N_{1}
$$

in the standard inner product

$$
\left\langle\Phi \mid \Phi^{\prime}\right\rangle=\int d^{D} x \Phi^{\dagger} \Phi^{\prime}=\frac{1}{2} \int d^{D} x\left(\phi^{*} \phi^{\prime}+\tilde{\phi}^{*} \tilde{\phi}^{\prime}\right),
$$

where $D$ is the number of spatial dimensions under consideration. Since there is a continuous exchange of atoms between the condensed and noncondensed phases, this norm will not be conserved in time. Indeed, this fact is already encoded in the evolution equation (10). Actually, it is straightforward to see that

$$
i \hbar \frac{d}{d t}\left\langle\Phi, \Phi^{\prime}\right\rangle=\left\langle\left(\mathcal{M}^{\dagger}-\mathcal{M}\right) \Phi, \Phi^{\prime}\right\rangle .
$$

However, the operator $\mathcal{M}$ is not self-adjoint in the positivedefinite inner product (14), but satisfies the following properties:

$$
\sigma_{x} \mathcal{M} \sigma_{x}=-\mathcal{M}^{*}, \quad \sigma_{z} \mathcal{M} \sigma_{z}=\mathcal{M}^{\dagger},
$$

and hence this inner product is not preserved in the evolution, as we have already mentioned.

These are the relevant properties of $\mathcal{M}$. In fact, in view of the last equality, we can introduce a "Bogolubov" inner product

$$
\left\langle\Phi \mid \Phi^{\prime}\right\rangle_{\mathrm{B}}=\int d^{D} x \Phi^{\dagger} \sigma_{z} \Phi^{\prime},
$$

in which $\mathcal{M}$ is self-adjoint. Indeed, it is straightforward to check that

$$
\left\langle\Phi \mid \mathcal{M} \Phi^{\prime}\right\rangle_{\mathrm{B}}=\left\langle\mathcal{M} \Phi \mid \Phi^{\prime}\right\rangle_{\mathrm{B}} .
$$

Therefore we see that the price to pay for making the evolution operator $\mathcal{M}$ self-adjoint is the introduction of an inner product $\langle\cdot \mid \cdot\rangle_{\mathrm{B}}$ which is not positive definite. Indeed, this Bogolubov inner product has the following properties:

(i) It is conserved in the evolution of the lab time $t$.

(ii) It is Hermitian, i.e., $\left\langle\Phi \mid \Phi^{\prime}\right\rangle_{\mathrm{B}}^{*}=\left\langle\Phi^{\prime} \mid \Phi\right\rangle_{\mathrm{B}}$.

(iii) It is antilinear in the first argument and linear in the second; i.e., for any complex number $\alpha$,

$$
\begin{aligned}
& \left\langle\alpha \Phi \mid \Phi^{\prime}\right\rangle_{\mathrm{B}}=\alpha^{*}\left\langle\Phi \mid \Phi^{\prime}\right\rangle_{\mathrm{B}}, \\
& \left\langle\Phi \mid \alpha \Phi^{\prime}\right\rangle_{\mathrm{B}}=\alpha\left\langle\Phi \mid \Phi^{\prime}\right\rangle_{\mathrm{B}} .
\end{aligned}
$$

(iv) It is not positive definite, since it satisfies

$$
\left\langle\sigma_{x} \Phi^{*} \mid \sigma_{x} \Phi^{\prime *}\right\rangle_{\mathrm{B}}=-\left\langle\Phi^{\prime} \mid \Phi\right\rangle_{\mathrm{B}} .
$$

Finally, note that the physical solutions, i.e. those that satisfy $\sigma_{x} \Phi^{*}=\Phi$ because of condition (12), have zero norm, as can easily be seen from Eq. (20).

\section{Mode expansion}

The evolution operator $\mathcal{M}$ is self-adjoint in a non-positive-definite inner product, and therefore it may have complex eigenvalues. We will assume that the condensate is stable, which implies that genuinely complex frequencies cannot be present. 
In view of the properties (16), it is easy to see that, if

$$
U_{k}=\frac{1}{\sqrt{2}}\left(\begin{array}{l}
u_{k} \\
v_{k}
\end{array}\right)
$$

is an eigenspinor of $\mathcal{M}$ with eigenvalue $\omega_{k}$, i.e., if

$$
\mathcal{M} U_{k}=\omega_{k} U_{k}
$$

then

$$
V_{k}^{*}=\sigma_{x} U_{k}^{*}=\frac{1}{\sqrt{2}}\left(\begin{array}{c}
v_{k}^{*} \\
u_{k}^{*}
\end{array}\right)
$$

is an eigenspinor of $\mathcal{M}$ with eigenvalue $-\omega_{k}$. Besides, $\sigma_{z} U_{k}$ is an eigenvector of $\mathcal{M}^{\dagger}$ with eigenvalue $\omega_{k}$. Furthermore, the modes $U_{k}$ and $V_{k}^{*}$ are orthogonal (and can be chosen orthonormal) in the Bogolubov inner product:

$$
\begin{gathered}
\left\langle U_{k} \mid U_{l}\right\rangle_{\mathrm{B}}=\frac{1}{2} \int d^{D} x\left(u_{k}^{*} u_{l}-v_{k}^{*} v_{l}\right)=\delta_{k l}, \\
\left\langle U_{k} \mid V_{l}^{*}\right\rangle_{\mathrm{B}}=\frac{1}{2} \int d^{D} x\left(u_{k}^{*} v_{l}^{*}-v_{k}^{*} u_{l}^{*}\right)=0, \\
\left\langle V_{k}^{*} \mid V_{l}^{*}\right\rangle_{\mathrm{B}}=\frac{1}{2} \int d^{D} x\left(v_{k} v_{l}^{*}-u_{k} u_{l}^{*}\right)=-\delta_{k l} .
\end{gathered}
$$

Any spinor $\Phi$ solution of Eq. (10) can be expanded in this basis:

$$
\Phi=\sum_{k}\left(a_{k} U_{k}+b_{k}^{*} V_{k}^{*}\right),
$$

and its norm is given by

$$
\langle\Phi \mid \Phi\rangle_{\mathrm{B}}=\sum_{k}\left(\left|a_{k}\right|^{2}-\left|b_{k}\right|^{2}\right) .
$$

We therefore again see that the physical solutions - those satisfying the condition (12)- have zero norm, since they satisfy $a_{k}=b_{k}$. Note that the modes themselves are not physical, not only because they may be generalized eigenvectors normalized to the Dirac delta, but also because, in general, they do not satisfy Eq. (12): $\sigma_{x} U_{k}^{*}=V_{k}^{*} \neq U_{k}$, as we have seen.

Finally, the number of field degrees of freedom carried by the spinor $\Phi$ is just two. Indeed, two complex (four real) functions are needed at an initial time to obtain the value of $\Phi$ at any other time. Condition (12), which ensures the physical nature of the configuration, reduces this number to one (two real initial functions), which is precisely the number of field degrees of freedom of a real relativistic scalar field. In the next section we will actually describe the condensate perturbations as a real scalar field satisfying (in the appropriate limit of long wavelengths) a relativistic wave equation.

\section{KLEIN-GORDON APPROACH}

As an alternative to the approach followed in the previous section, we can linearize the continuity [(3)] and Bernoulli [(4)] equations around a background condensate characterized by $n_{0}$ and $\theta_{0}$. Let us introduce the density $\tilde{n}_{1}$ and phase $\theta_{1}$ perturbations:

$$
n=n_{0}+g^{-1} \tilde{n}_{1}, \quad \theta=\theta_{0}+\theta_{1} .
$$

These perturbations obey the equations

$$
\begin{gathered}
\partial_{t} \tilde{n}_{1}+\nabla \cdot\left(\tilde{n}_{1} \mathbf{v}+c^{2} \nabla \theta_{1}\right)=0, \\
\partial_{t} \theta_{1}+\mathbf{v} \cdot \nabla \theta_{1}+(1-\Theta) \tilde{n}_{1}=0,
\end{gathered}
$$

where $\Theta$ is the operator

$$
\Theta=\frac{1}{4} \xi^{2} \nabla\left[c^{2} \cdot \nabla\left(c^{-2} \star\right)\right],
$$

$\xi=\hbar /(m c)$ is the healing length, and the $\star$ stands for the argument upon which $\Theta$ acts.

Note that the perturbation fields $\phi, \tilde{\phi}$ introduced in the previous sections are related to the complexified density $\tilde{n}_{1}$ and phase $\theta_{1}$ perturbations in the following way:

$$
\begin{aligned}
& \phi=e^{i \theta_{0} / \hbar} \frac{1}{\sqrt{g m}}\left(\frac{1}{2 c} \tilde{n}_{1}+i \frac{1}{\xi} \theta_{1}\right), \\
& \tilde{\phi}=e^{-i \theta_{0} / \hbar} \frac{1}{\sqrt{g m}}\left(\frac{1}{2 c} \tilde{n}_{1}-i \frac{1}{\xi} \theta_{1}\right),
\end{aligned}
$$

in terms of which

$$
\begin{aligned}
& \tilde{n}_{1}=c \sqrt{g m}\left(e^{-i \theta_{0} / \hbar} \phi+e^{i \theta_{0} / \hbar} \tilde{\phi}\right), \\
& \theta_{1}=-i \frac{\xi}{2} \sqrt{g m}\left(e^{-i \theta_{0} / \hbar} \phi-e^{i \theta_{0} / \hbar} \tilde{\phi}\right) .
\end{aligned}
$$

The condition (12) that $\phi$ and $\tilde{\phi}$ represent a physical solution to the Bogolubov equation (5) translates into reality conditions for $\tilde{n}_{1}$ and $\theta_{1}$. It is also interesting to note that the commutation relation for these two fields is

$$
\left[\hat{\tilde{n}}_{1}(\mathbf{x}, t), \hat{\theta}_{1}\left(\mathbf{x}^{\prime}, t\right)\right]=i g \hbar \delta\left(\mathbf{x}-\mathbf{x}^{\prime}\right),
$$

as a direct consequence of the commutation relation (8) for $\hat{\phi}$. Thus, $\tilde{n}_{1}$ and $\theta_{1}$ are canonically conjugate fields.

\section{A. Generalized Klein-Gordon equation}

We can now combine the two equations (30) and (31) for $\theta_{1}$ and $\tilde{n}_{1}$ in order to obtain a second order (in time) differential equation for $\theta_{1}$. More explicitly, we can obtain $\tilde{n}_{1}$ as a function of $\theta_{1}$ from Eq. (31) by formally inverting the operator $(1-\Theta)$ :

$$
\tilde{n}_{1}=-\mathcal{W}\left(\partial_{t}+\mathbf{v} \cdot \nabla\right) \theta_{1},
$$

where

$$
\mathcal{W}=(1-\Theta)^{-1}=\sum_{n=0}^{\infty} \Theta^{n}
$$


Note that generally this is a well-defined procedure because of the negativity of the operator $\Theta$. Indeed, for homogeneous profiles, $\Theta$ is proportional to the Laplacian, which is obviously a negative operator. As long as the profile is sufficiently smooth such that $c$ is slowly varying on the healing length scale, this negative character will not be altered. Relevant departures from this behavior would require profiles whose densities vary significantly within length scales comparable with the healing length, which is not only easily avoidable in practice but might actually be very hard to realize. Keeping these comments in mind, we can insert Eq. (36) into Eq. (30) to obtain the single equation

$$
-\left[\partial_{t}+\nabla \cdot(\mathbf{v} \star)\right] \mathcal{W}\left(\partial_{t}+\mathbf{v} \cdot \nabla\right) \theta_{1}+\nabla \cdot\left(c^{2} \nabla \theta_{1}\right)=0 .
$$

This equation is a higher order differential equation which generalizes the Klein-Gordon equation.

In the limit $\mathcal{W} \rightarrow 1$, attained when the gradients in $\theta_{1}$ are relevant only for length scales much larger than the healing length, a proper Klein-Gordon equation is recovered. Indeed, in this case, this evolution equation can be written in the form [4]

$$
\partial_{\mu}\left(\sqrt{-g} g^{\mu \nu} \partial_{\nu} \theta_{1}\right)=0,
$$

where $g_{\mu \nu}$ is the acoustic metric

$$
g_{\mu \nu}=c^{2 /(D-1)}\left(\begin{array}{cc}
-\left(c^{2}-\mathbf{v}^{2}\right) & -\mathbf{v}^{t} \\
-\mathbf{v} & \mathbb{1}
\end{array}\right) .
$$

The corresponding Klein-Gordon inner product can be written as

$$
\begin{aligned}
\left\langle\theta_{1} \mid \theta_{1}^{\prime}\right\rangle_{\mathrm{KG}} & =i \int d^{D} x \sqrt{q} \theta_{1}^{*} \stackrel{\leftrightarrow}{\partial}_{n} \theta_{1}^{\prime} \\
& =i \int d^{D} x \theta_{1}^{*}\left(\overleftrightarrow{\partial_{t}+\mathbf{v} \cdot \nabla}\right) \theta_{1}^{\prime},
\end{aligned}
$$

where $\sqrt{q}=c^{D /(D-1)}$ is the determinant of the metric in the spatial slice $t=$ constant, $n^{\mu}=c^{-D /(D-1)}(1, \mathbf{v})$ is its normal, and $\partial_{n}=n^{\mu} \partial_{\mu}$.

\section{B. Generalized Klein-Gordon product}

In the general case, we can introduce an inner product that generalizes the Klein-Gordon product by taking into account that the time derivative term is now modified by the operator $\mathcal{W}$. In $D$ spatial dimensions, this $\mathcal{W}$-KleinGordon inner product turns out to be

$$
\left\langle\theta_{1} \mid \theta_{1}^{\prime}\right\rangle_{\mathcal{W}-\mathrm{KG}}=i \int d^{D} x \theta_{1}^{*}\left[\widetilde{\mathcal{W}\left(\partial_{t}+\mathbf{v} \cdot \vec{\nabla}\right)}\right] \theta_{1}^{\prime} .
$$

It is clear from this expression that it reduces to the standard relativistic Klein-Gordon product in the limit $\mathcal{W} \rightarrow 1$.

It should be stressed that the operator $\mathcal{W}$ breaks the local Lorentz invariance of the Klein-Gordon equation.
This means that an effective curved-spacetime geometry is recovered only for condensate perturbations such that $\mathcal{W}$ is very close to 1 . Furthermore, as we will see below, the dispersion relation for this $\mathcal{W}$-Klein-Gordon equation is the same as that for the Klein-Gordon equation modified with fourth-order spatial derivatives. Here, however, the Klein-Gordon inner product is modified in each $t=$ constant slice by the action of the operator $\mathcal{W}$. The Klein-Gordon equation modified with fourth-order spatial derivatives, on the other hand, shares the Klein-Gordon product in each $t=$ constant slice (41) with the proper Klein-Gordon equation [16].

In spite of these modifications, the $\mathcal{W}$-Klein-Gordon product (42) shares the following properties with the standard Klein-Gordon product:

(i) It is conserved in the lab time $t$. However, unlike the Klein-Gordon product, which is conserved in any inertial time, the $\mathcal{W}$-Klein-Gordon product is conserved only in the lab time. This is a logical consequence of the fact that local Lorentz invariance is no longer in operation.

(ii) It is Hermitian, i.e., $\left\langle\theta_{1} \mid \theta_{1}^{\prime}\right\rangle_{\mathcal{W} \text {-KG }}^{*}=\left\langle\theta_{1}^{\prime} \mid \theta_{1}\right\rangle_{\mathcal{W} \text {-KG }}$.

(iii) It is antilinear in the first argument and linear in the second.

(iv) It is not positive definite. Indeed,

$$
\left\langle\theta_{1}^{*} \mid \theta_{1}^{\prime *}\right\rangle_{\mathcal{W}-\mathrm{KG}}=-\left\langle\theta_{1}^{\prime} \mid \theta_{1}\right\rangle_{\mathcal{W}-\mathrm{KG}} .
$$

(v) There exists at least one basis $\left\{\theta_{1 i}\right\}$ of orthonormal solutions such that

$$
\begin{gathered}
\left\langle\theta_{1 i} \mid \theta_{1 j}\right\rangle_{\mathcal{W}-\mathrm{KG}}=\delta_{i j}, \quad\left\langle\theta_{1 i} \mid \theta_{1 j}^{*}\right\rangle_{\mathcal{W}-\mathrm{KG}}=0, \\
\left\langle\theta_{1 i}^{*} \mid \theta_{1 j}^{*}\right\rangle_{\mathcal{W}-\mathrm{KG}}=-\delta_{i j} .
\end{gathered}
$$

(vi) The norm of any solution $\theta_{1}=\sum_{i}\left(a_{i} \theta_{1 i}+b_{i}^{*} \theta_{1 i}^{*}\right)$ is

$$
\left\langle\theta_{1} \mid \theta_{1}\right\rangle_{\mathcal{W}-\mathrm{KG}}=\sum_{i}\left(\left|a_{i}\right|^{2}-\left|b_{i}\right|^{2}\right) .
$$

This norm vanishes for real scalar fields such as the physical phase perturbation.

At this stage, it is worth noting that the $\mathcal{W}$-KleinGordon inner product has the same properties that we stated above for the Bogolubov inner product. In fact, we will see that these two products are indeed equivalent.

\section{Mode expansion}

We will now discuss the form of the modes and their normalization. For the sake of simplicity and definiteness, let us concentrate on a case of particular interest, namely, the case of a background profile which becomes time independent in the asymptotic future. Then we can try an ansatz of the form $A e^{-i \omega u(t, \mathbf{x})}$, which in the pure onedimensional Klein-Gordon case leads to exact orthonormal modes $(2 c|k|)^{-1 / 2} e^{-i(\omega t-k x)}$. 
Let us define

$$
\bar{\omega}(t, \mathbf{x})=\omega \partial_{t} u, \quad \mathbf{k}(t, \mathbf{x})=-\omega \nabla u .
$$

The condition that the profile becomes stationary in the asymptotic future implies that $\bar{\omega}(t \rightarrow \infty, \mathbf{x})=\omega$ and that $\mathbf{k}(t, \mathbf{x})$ is time independent in this limit. Then $A e^{-i \omega u}$ is an approximate solution which can be found in the regime where $\mathbf{k}, \bar{\omega}$, and $A$ are slowly varying functions (in space and time). Introduction of this approximate solution into the modified Klein-Gordon equation yields, to lowest order, the dispersion relation

$$
(\bar{\omega}-\mathbf{v} \cdot \mathbf{k})^{2}=c^{2} \mathbf{k}^{2} \Gamma_{\mathbf{k}}^{2},
$$

and hence the form of $u(t, \mathbf{x})$, where

$$
\Gamma_{\mathbf{k}}=+\sqrt{1+\xi^{2} \mathbf{k}^{2} / 4}
$$

The next-to-lowest order yields the prefactor $A$ so that a complete set of approximate modes is given by

$$
\theta_{1 \mathbf{k}}=\frac{\sqrt{\Gamma_{\mathbf{k}}}}{\sqrt{4 \pi c|\mathbf{k}|}} e^{-i \omega u}
$$

The next order provides information about the spread of these modes in the directions perpendicular to that of group propagation. The relativistic limit $\mathcal{W} \rightarrow 1$ obviously corresponds to $\Gamma_{\mathbf{k}} \rightarrow 1$, i.e., to the long-wavelength limit $\xi|\mathbf{k}| \ll 1$, which gives rise to the well-known KleinGordon modes. These approximate modes are orthonormal (in the same level of approximation) in the $\mathcal{W}$-KleinGordon product (42) as can be seen by evaluating it at $t \rightarrow$ $\infty$. Evaluated at any other finite time $t$, small deviations which are consistent with the level of approximation that we are using may appear.

As mentioned above, any real solution $\theta_{1}$ can be written as a linear combination of these modes,

$$
\theta_{1}=\sum_{\mathbf{k}}\left(a_{\mathbf{k}} \theta_{1 \mathbf{k}}+a_{\mathbf{k}}^{*} \theta_{1 \mathbf{k}}^{*}\right)
$$

and therefore has zero norm.

For completeness, it is also straightforward to check from Eq. (36) that

$$
\tilde{n}_{1 \mathbf{k}}=i \frac{c|\mathbf{k}|}{\Gamma_{\mathbf{k}}} \theta_{1 \mathbf{k}} .
$$

Finally, before examining the relation between the Bogolubov and the Klein-Gordon formalisms, we mention the existence of a third alternative approach [17], which leads to a genuinely complex differential equation of second order in time and fourth order in space for the field perturbation $\phi$. Indeed, if we write $\phi^{*}$ in terms of $\phi$ from the Bogolubov equation and use this expression in the complex conjugate of this same equation, we obtain

$$
\begin{aligned}
\left\{\left[\hbar\left(\partial_{t}+\mathbf{v} \cdot \nabla\right)-i T_{\rho}\right] \frac{1}{c^{2}}\left[\hbar\left(\partial_{t}+\mathbf{v} \cdot \nabla\right)+i T_{\rho}\right]\right. & \left.+2 m T_{\rho}\right\} \varphi \\
& =0, \quad(52)
\end{aligned}
$$

where $\varphi=\phi / \psi_{0}$ and $T_{\rho}=-\frac{\hbar^{2}}{2 m c^{2}} \nabla \cdot\left(c^{2} \nabla \star\right)$. It might seem at first sight that the number of degrees of freedom is equivalent to that of a complex scalar field (i.e., two sets of complex Fourier coefficients). However, $\varphi$ and $\varphi^{*}$ are not independent but are related by the Bogolubov equation itself, leaving — as before- just one field degree of freedom (a single set of complex Fourier coefficients), and, in fact, the scalar product in the space of solutions of this equation is the Bogolubov one.

\section{EQUIVALENCE BETWEEN BOTH PRODUCTS}

The following question of interest regards the relation between the Bogolubov product and the $\mathcal{W}$-Klein-Gordon product introduced in the previous sections. More specifically, what is the relation between the concepts of quasiparticle and antiquasiparticle in both formalisms?

Let us consider two spinors $\Phi$ and $\Phi^{\prime}$, solutions to the Bogolubov evolution equation (10). Then, taking into account the relation (33) between both representations, i.e., between the perturbation fields $\phi, \tilde{\phi}$ and $\tilde{n}_{1}, \theta_{1}$, we obtain

$$
\begin{aligned}
\left\langle\Phi \mid \Phi^{\prime}\right\rangle_{\mathrm{B}} & =\frac{1}{2} \int d^{D} x\left(\phi^{*} \phi^{\prime}-\tilde{\phi}^{*} \tilde{\phi}^{\prime}\right) \\
& =\frac{i}{2 g \hbar} \int d^{D} x\left(\tilde{n}_{1}^{*} \theta_{1}^{\prime}-\theta_{1}^{*} \tilde{n}_{1}^{\prime}\right) .
\end{aligned}
$$

Finally, the relation (36) between the density and phase perturbation allows us to write this Bogolubov product as

$$
\begin{gathered}
\left\langle\Phi \mid \Phi^{\prime}\right\rangle_{\mathrm{B}}=\frac{i}{2 g \hbar} \int d^{D} x \theta_{1}^{*}\left[\stackrel{\mathcal{W}\left(\partial_{t}+\mathbf{v} \cdot \vec{\nabla}\right)}{ }\right] \theta_{1}^{\prime} \\
=\frac{1}{2 g \hbar}\left\langle\theta_{1} \mid \theta_{1}^{\prime}\right\rangle_{\mathcal{W}-\mathrm{KG}} .
\end{gathered}
$$

So, we see that the Bogolubov and the $\mathcal{W}$-Klein-Gordon products are indeed equivalent.

Also, provided a set of orthonormal modes $\theta_{1 \mathrm{k}}$ of the $\mathcal{W}$-Klein-Gordon equation, it is straightforward to construct an orthonormal set of modes for the Bogolubov equation (10) by means of the relations (33) and (51):

$$
\begin{aligned}
& u_{\mathbf{k}}=e^{i \theta_{0} / \hbar} \frac{1}{\sqrt{g m} \xi}\left(1+\frac{\xi|\mathbf{k}|}{2 \Gamma_{\mathbf{k}}}\right) \theta_{1 \mathbf{k}}, \\
& v_{\mathbf{k}}=e^{-i \theta_{0} / \hbar} \frac{1}{\sqrt{g m} \xi}\left(-1+\frac{\xi|\mathbf{k}|}{2 \Gamma_{\mathbf{k}}}\right) \theta_{1 \mathbf{k}} .
\end{aligned}
$$

Therefore positive (resp. negative) norm modes in the $\mathcal{W}$ Klein-Gordon product are mapped to positive (resp. negative) norm modes in the Bogolubov product and vice versa. This means that, whether we choose to analyze quasiparticle creation processes (e.g., Hawking radiation in a black 
hole configuration) in a modified relativistic framework such as in [18] or a condensed-matter context such as in [17], the results should coincide. The reason for this coincidence is that, as we have seen, there is a one-to-one relation between both formalisms, and the concepts of quasiparticle and antiquasiparticle, as well as the ambiguities inherent in these definitions (which are related to the observer dependence of the concepts involved), are the same in both formalisms.

\section{THE CHOICE OF VACUUM STATE}

One can now proceed with the quantization of the system following the standard Fock procedure. One only needs to find an orthonormal mode basis to expand the field operators that characterize the quantum perturbations. In this manner, one can define creation and annihilation operators and a vacuum state for the system. The fact that the inner product is not positive definite tells us that the selection of a specific set of positive (negative) norm modes can be done in many different ways. To illustrate the procedure, let us consider a one-dimensional stationary flow in the condensate, which simulates the presence of a black hole, but with the internal singularity substituted by a second asymptotic region [19]. Among the different sets of modes that can be selected, there are three of special relevance, which we will call the "in" set, the "out" set, and the "stationary" set, whose meaning will be discussed shortly. Each positive energy mode of each set can be characterized by its frequency (which is invariant due to the stationarity of the system) and by a discrete label which, depending on the frequency, can acquire the values 1, 2 or 1, 2, 3 (see the discussion in [20] regarding the number of normalizable modes in different configurations).

We will now classify these modes, using the following subscripts. The $p$ and $f$ subscripts represent "past" and "future" (in a scattering process), while $u$ and $w$ represent the "right-going" and "left-going" character of wave packets centered around the particular mode frequency with respect to the lab.

Let us consider the dispersion relation (47) formally as a relation between $\omega, k$, and $x$ [through $v(x)$ ]. Then, for any given real frequency $\omega$ and any point $x$ in the configuration, the dispersion relation may have two or four real roots $k$; see Fig. 1. Actually, there exists a critical frequency $\omega_{c}$ such that, for $\omega>\omega_{c}$, independently of $x$, there always exist two real roots only, one positive and one negative. We denote by $k_{u p}$ the positive root when $x \rightarrow-\infty$, by $k_{w p}$ the absolute value of the negative root when $x \rightarrow+\infty$, by $k_{u f}$ the positive root when $x \rightarrow+\infty$, and finally by $k_{w f}$ the absolute value of the negative root when $x \rightarrow-\infty$. For $0<$ $\omega<\omega_{c}$, depending on $x$, there can be either two or four roots (there is a critical position at which there are just three solutions; here we will not discuss this critical situation). Two of the roots are always equivalent to the

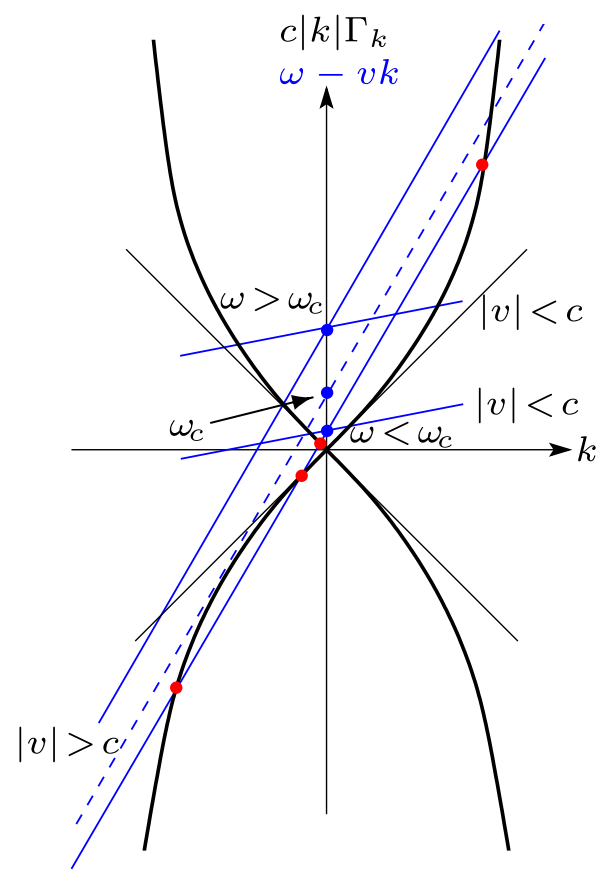

FIG. 1 (color online). Dispersion relation (47) for various values of $\omega$ and of $|v|$, scaled at $c=1$. The intersection points between the line $\omega-v k$ (in blue) and the various branches of the dispersion relation (in black) mark the real (normalizable) mode solutions for a given $\omega$. The critical frequency $\omega_{c}$ represents the frequency at which additional, "extraordinary" roots appear for the maximal $|v|$ attained in the configuration. Then, for $\omega>\omega_{c}$, there are always only two normalizable solutions. For $\omega<\omega_{c}$, there can be either two or four normalizable solutions, depending on the value of $|v|$.

previous ones; we will use the same notation for them. In the case of the existence of four real roots, the two additional ones always correspond to negative values of $k$. We will call these additional modes "extraordinary" because they are absent in the subsonic regime. Let us then denote by $k_{e p}$ the absolute value of the most negative extraordinary root when $x \rightarrow-\infty$ and by $k_{e f}$ the absolute value of the second most negative extraordinary root when $x \rightarrow$ $-\infty$. The corresponding wave packets are right-going $\left(k_{e p}\right)$ and left-going $\left(k_{e f}\right)$, respectively.

We summarize this classification in Table I.

TABLE I. Classification of normalizable mode solutions (real roots) in the asymptotic regions. The arrows indicate the left- or right-going character of the corresponding wave packets with respect to the lab.

\begin{tabular}{lllll}
\hline \hline & \multicolumn{2}{c}{$t \rightarrow-\infty$} & \multicolumn{2}{c}{$t \rightarrow+\infty$} \\
& $\longrightarrow$ & $\longleftarrow$ & $\longrightarrow$ & $\longleftarrow$ \\
\hline$x \rightarrow+\infty(v<c)$ & & $k_{w p}$ & $k_{u f}$ & \\
$x \rightarrow-\infty(v>c)$ and $\omega>\omega_{c}$ & $k_{u p}$ & & & $k_{w f}$ \\
$x \rightarrow-\infty(v>c)$ and $\omega<\omega_{c}$ & $k_{u p}$ & & & $k_{w f}$ \\
& $k_{e p}$ & & $k_{e f}$ \\
\hline \hline
\end{tabular}


Given the equivalence displayed in the previous section, we are free to describe the perturbations either by a spinor in the Bogolubov formulation or by the phase perturbation operator $\hat{\theta}$ in the Klein-Gordon formulation. For notational simplicity, we omit the subscript 1 for the perturbations in this section.

\section{A. In vacuum state}

It can be shown [20] that the positive energy modes that constitute the in set (such that, in the in vacuum defined by them, there are no quasiparticles in the asymptotic past) can be described as follows, up to a global mode-dependent normalization constant:

(i) For $\omega>\omega_{c}$,

$$
\begin{aligned}
& \theta_{\omega, 1}^{\text {in }} \stackrel{x \rightarrow-\infty}{\longrightarrow} e^{-i \omega t}\left(e^{i k_{u p} x}+\tilde{R}_{\omega}^{\text {in }} e^{-i k_{w f} x}\right), \\
& \theta_{\omega, 1}^{\text {in }} \stackrel{x \rightarrow+\infty}{\longrightarrow} e^{-i \omega t}\left(\tilde{T}_{\omega}^{\text {in }} e^{i k_{u f} x}\right) ; \\
& \theta_{\omega, 2}^{\text {in }} \stackrel{x \rightarrow+\infty}{\longrightarrow} e^{-i \omega t}\left(e^{-i k_{w p} x}+R_{\omega}^{\text {in }} e^{i k_{u f} x}\right), \\
& \theta_{\omega, 2}^{\text {in }} \stackrel{x \rightarrow-\infty}{\longrightarrow} e^{-i \omega t}\left(T_{\omega}^{\text {in }} e^{-i k_{w f} x}\right) .
\end{aligned}
$$

(ii) For $\omega<\omega_{c}$,

$$
\begin{aligned}
& \theta_{\omega, 1}^{\text {in }} \stackrel{x \rightarrow-\infty}{\longrightarrow} e^{-i \omega t}\left(e^{i k_{u p} x}+\tilde{R}_{\omega}^{\mathrm{in}} e^{-i k_{w f} x}+\tilde{R}_{\omega, e}^{\mathrm{in}} e^{-i k_{e f} x}\right), \\
& \theta_{\omega, 1}^{\text {in }} \stackrel{x \rightarrow+\infty}{\longrightarrow} e^{-i \omega t}\left(\tilde{T}_{\omega}^{\mathrm{in}} e^{i k_{u f} x}\right) ; \\
& \theta_{\omega, 2}^{\text {in }} \stackrel{x \rightarrow+\infty}{\longrightarrow} e^{-i \omega t}\left(e^{-i k_{w p} x}+R_{\omega}^{\text {in }} e^{i k_{u f} x}\right), \\
& \theta_{\omega, 2}^{\text {in }} \stackrel{x \rightarrow-\infty}{\longrightarrow} e^{-i \omega t}\left(T_{\omega}^{\mathrm{in}} e^{-i k_{w f} x}+T_{\omega, e}^{\text {in }} e^{-i k_{e f} x}\right) \\
& \theta_{\omega, 3}^{\text {in }} \stackrel{x \rightarrow-\infty}{\longrightarrow} e^{i \omega t}\left(e^{-i k_{e p} x}+\bar{R}_{\omega}^{\mathrm{in}} e^{i k_{w f} x}+\bar{R}_{\omega, e}^{\text {in }} e^{i k_{e f} x}\right) \\
& \theta_{\omega, 3}^{\text {in }} \stackrel{x \rightarrow+\infty}{\longrightarrow} e^{i \omega t}\left(\bar{T}_{\omega} e^{-i k_{u f} x}\right),
\end{aligned}
$$

where the $R$ 's and $T$ 's are mode-dependent reflection and transmission coefficients. In other words, every mode behaves in the specific form displayed by the above formulas in the appropriate asymptotic limits. These modes can be pictorially described as representing elementary scattering processes (see Fig. 2). For $\omega>\omega_{c}$ there are two independent and orthonormal scattering processes: (1) the scattering of an incoming wave from the left and (2) the scattering of an incoming wave from the right. For $0<\omega<\omega_{c}$ there are three independent and orthonormal scattering processes: $\left(1^{\prime}\right)$ an incoming wave from the left, $\left(2^{\prime}\right)$ an incoming wave from the right, and $\left(3^{\prime}\right)$ an incoming extraordinary wave from the left. All these three processes end up being a combination of three outgoing waves at future infinity, one of them extraordinary. Notice that $\omega$ in the exponential of the mode $\theta_{\omega, 3}^{\text {in }}$ appears with a positive
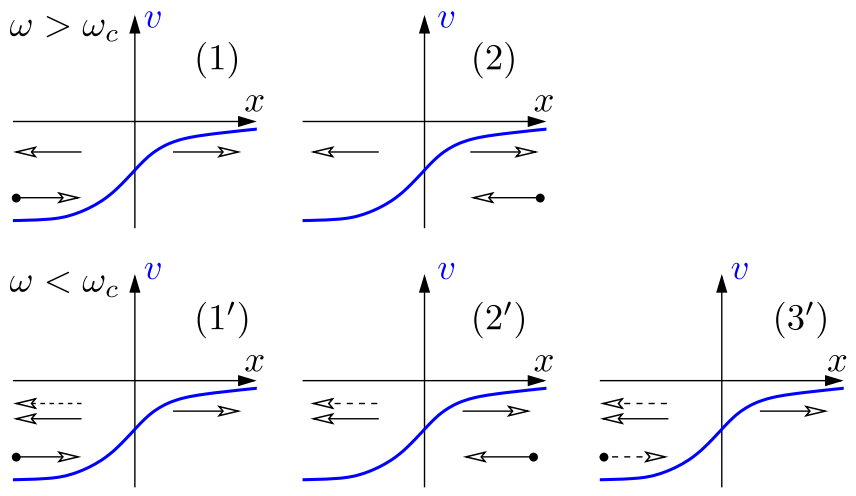

FIG. 2 (color online). The in basis. $\omega>\omega_{c}$ : (1) represents a scattering process in which there is an incoming wave from the left; (2) represents a scattering process in which there is an incoming wave from the right. $0<\omega<\omega_{c}$ : $\left(1^{\prime}\right)$ represents a scattering process in which there is an incoming wave from the left; $\left(2^{\prime}\right)$ represents a scattering process in which there is an incoming wave from the right; (3') represents a scattering process in which there is an extraordinary incoming wave from the left. Solid arrows represent ordinary modes, while dashed arrows represent extraordinary modes.

sign, contrarily to the negative sign in the other modes. These signs are chosen in order to define positive energy (norm) modes. ${ }^{1}$

\section{B. Out vacuum state}

In an equivalent way, one can construct the positive energy out basis (such that, in the out vacuum defined by it, there are no quasiparticles in the asymptotic future) [20], up to a global mode-dependent normalization constant:

(I) For $\omega>\omega_{c}$,

$$
\begin{aligned}
& \theta_{\omega, 1}^{\text {out }} \stackrel{x \rightarrow+\infty}{\longrightarrow} e^{-i \omega t}\left(e^{i k_{u f} x}+R_{\omega}^{\text {out }} e^{-i k_{w p} x}\right), \\
& \theta_{\omega, 1}^{\text {out }} \stackrel{x \rightarrow-\infty}{\longrightarrow} e^{-i \omega t}\left(T_{\omega}^{\text {out }} e^{i k_{u p} x}\right) ; \\
& \theta_{\omega, 2}^{\text {out }} \stackrel{x \rightarrow-\infty}{\longrightarrow} e^{-i \omega t}\left(e^{-i k_{w f} x}+\tilde{R}_{\omega}^{\text {out }} e^{i k_{u p} x}\right), \\
& \theta_{\omega, 2}^{\text {out }} \stackrel{x \rightarrow+\infty}{\longrightarrow} e^{-i \omega t}\left(\tilde{T}_{\omega}^{\text {out }} e^{-i k_{w p} x}\right) .
\end{aligned}
$$

(II) For $0<\omega<\omega_{c}$,

$$
\begin{gathered}
\theta_{\omega, 1}^{\text {out }} \stackrel{x \rightarrow+\infty}{\longrightarrow} e^{-i \omega t}\left(e^{i k_{u f} x}+R_{\omega}^{\text {out }} e^{-i k_{w p} x}\right) \\
\theta_{\omega, 1}^{\text {out }} \stackrel{x \rightarrow-\infty}{\longrightarrow} e^{-i \omega t}\left(T_{\omega}^{\text {out }} e^{i k_{u p} x}+T_{\omega, e}^{\text {out }} e^{i k_{e p} x}\right) \\
\theta_{\omega, 2}^{\text {out }} \stackrel{x \rightarrow-\infty}{\longrightarrow} e^{-i \omega t}\left(e^{-i k_{w f} x}+\tilde{R}_{\omega}^{\text {out }} e^{i k_{u p} x}+\tilde{R}_{\omega, e}^{\text {out }} e^{i k_{e p} x}\right), \\
\theta_{\omega, 2}^{\text {out }} \stackrel{x \rightarrow+\infty}{\longrightarrow} e^{-i \omega t}\left(\tilde{T}_{\omega}^{\text {out }} e^{-i k_{w p} x}\right)
\end{gathered}
$$

\footnotetext{
${ }^{1}$ In the notation of Ref. [20] our $\theta_{\omega, 3}^{\text {in }}$ corresponds to $\varphi_{-\omega}^{u}$.
} 

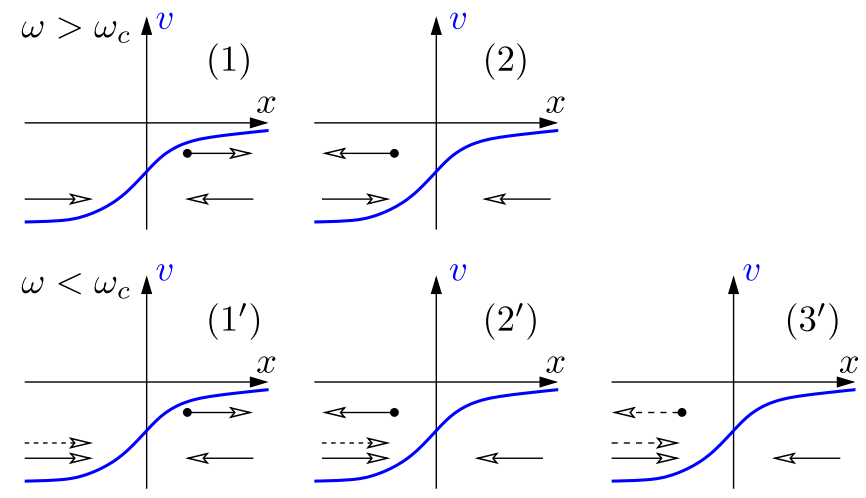

FIG. 3 (color online). The out basis. The interpretation is as in (time-reversed) Fig. 2.

$$
\begin{aligned}
& \theta_{\omega, 3}^{\text {out }} \stackrel{x \rightarrow-\infty}{\longrightarrow} e^{i \omega t}\left(e^{i k_{e f} x}+\bar{R}_{\omega}^{\text {out }} e^{-i k_{u p} x}+\bar{R}_{\omega, e}^{\text {out }} e^{-i k_{e p} x}\right) \\
& \theta_{\omega, 3}^{\text {out }} \stackrel{x \rightarrow+\infty}{\longrightarrow} e^{i \omega t}\left(\bar{T}_{\omega}^{\text {out }} e^{i k_{w p} x}\right)
\end{aligned}
$$

The pictorial representations of these elementary scattering processes can be seen in Fig. 3. They correspond to the elementary scattering processes of Fig. 2 running backwards in time (note that for this identification we have to exchange labels 1 and 2).

For the case $\omega<\omega_{c}$, it is not possible to express the positive norm mode $\theta_{\omega, 1}^{\text {out }}$ only in terms of the positive norm modes $\theta_{\omega, 1}^{\text {in }}, \theta_{\omega, 2}^{\text {in }}, \theta_{\omega, 3}^{\text {in }}$. One has to use the negative norm modes $\theta_{\omega, 1}^{\text {in } *}, \theta_{\omega, 2}^{\text {in } *}, \theta_{\omega, 3}^{\text {in* }}$ as well. Therefore, the in vacuum state defined through the requirement $a_{\omega, i}^{\text {in }}\left|0_{\text {in }}\right\rangle=0$ contains quasiparticles coming out at the right asymptotic region. This phenomenon is usually called mode mixing and has been thoroughly analyzed in [20].

\section{Stationary vacuum state}

Let us finally describe the stationary set. As a preliminary stage, let us choose the following:

(i) For $\omega>\omega_{c}$,

$$
\theta_{\omega, 1}^{\mathrm{st}-\mathrm{p}}=\theta_{\omega, 1}^{\text {out }} \quad \text { and } \quad \theta_{\omega, 2}^{\mathrm{st}-\mathrm{p}}=\theta_{\omega, 2}^{\mathrm{in}} .
$$

(ii) For $0<\omega<\omega_{c}$,

$$
\theta_{\omega, 1}^{\mathrm{st}-\mathrm{p}}=\theta_{\omega, 1}^{\text {out }}, \quad \theta_{\omega, 2}^{\mathrm{st}-\mathrm{p}}=\theta_{\omega, 2}^{\text {in }},
$$

and the additional mode $\theta_{\omega, 3}^{\mathrm{st}-\mathrm{p}}$ given by

$$
\begin{aligned}
& \theta_{\omega, 3}^{\mathrm{st}-\mathrm{p}} \stackrel{x \rightarrow-\infty}{\longrightarrow} e^{-i \omega t}\left(A_{\omega} e^{i k_{u p} x}+A_{\omega, e} e^{i k_{e p} x}+B_{\omega} e^{-i k_{w f} x}\right. \\
&\left.+B_{\omega, e} e^{-i k_{e f} x}\right) \\
& \theta_{\omega, 3}^{\mathrm{st}-\mathrm{p}} \stackrel{x \rightarrow+\infty}{\longrightarrow} 0
\end{aligned}
$$

if it has a positive norm, or by
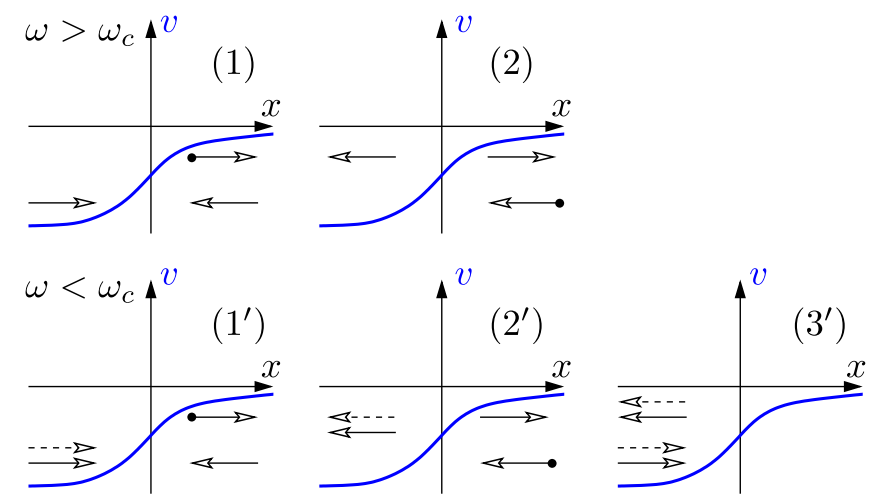

FIG. 4 (color online). Preliminary stationary basis. $\omega>\omega_{c}$ : (1) represents a scattering process in which there is an outgoing wave to the right; (2) represents a scattering process in which there is an incoming wave from the right. $0<\omega<\omega_{c}$ : $\left(1^{\prime}\right)$ represents a scattering process in which there is an outgoing wave to the right; $\left(2^{\prime}\right)$ represents a scattering process in which there is an incoming wave from the right; (3') represents a scattering process in which there are neither incoming nor outgoing waves on the right. These modes are not orthogonal. The stationary orthonormal basis is obtained by linear combinations of these modes. Solid arrows represent ordinary modes, while dashed arrows represent extraordinary modes.

$$
\begin{aligned}
& \theta_{\omega, 3}^{\mathrm{st}-\mathrm{p}} \stackrel{x \rightarrow-\infty}{\longrightarrow} e^{i \omega t}\left(A_{\omega} e^{-i k_{u p} x}+A_{\omega, e} e^{-i k_{e p} x}+B_{\omega} e^{i k_{w f} x}\right. \\
&\left.+B_{\omega, e} e^{i k_{e f} x}\right) \\
& \theta_{\omega, 3}^{\mathrm{st}-\mathrm{p}} \stackrel{x \rightarrow-\infty}{\longrightarrow}
\end{aligned}
$$

if it occurs that the conjugate of the expression in Eq. (69) is the one with a positive norm (this will depend on the specific shape of the velocity profile). The ratio $A_{\omega} / A_{\omega, e}$ is fixed by the condition that the scattering leads to no transmission in the right asymptotic region. Then, for a given $A_{\omega}, B_{\omega}$ and $B_{\omega, e}$ become fixed. Finally the value of $A_{\omega}$ itself can be fixed by requiring that the mode be normalized. Figure 4 shows a pictorial representation of these modes.

The previous three modes are clearly independent and normalized, but they are not orthogonal. However, starting from them, it is easy to define three new modes which are orthonormal. Consider the transformation

$$
\left(\begin{array}{c}
\theta_{\omega, 1}^{\mathrm{st}} \\
\theta_{\omega, 2}^{\mathrm{st}} \\
\theta_{\omega, 3}^{\mathrm{st}}
\end{array}\right)=\left(\begin{array}{ccc}
M_{11} & M_{12} & M_{13} \\
0 & M_{22} & M_{23} \\
0 & 0 & M_{33}
\end{array}\right)\left(\begin{array}{c}
\theta_{\omega, 1}^{\mathrm{st}-\mathrm{p}} \\
\theta_{\omega, 2}^{\mathrm{st}-\mathrm{p}} \\
\theta_{\omega, 3}^{\mathrm{st}-\mathrm{p}}
\end{array}\right),
$$

with

$$
M_{11}=N^{-1 / 2} \text {, }
$$

$$
M_{12}=N^{-1 / 2}\left(p_{23} p_{13}^{*}-p_{12}^{*}\right)\left(1-\left|p_{23}\right|^{2}\right)^{-1},
$$




$$
\begin{gathered}
M_{13}=N^{-1 / 2}\left(p_{12}^{*} p_{23}^{*}-p_{13}^{*}\right)\left(1-\left|p_{23}\right|^{2}\right)^{-1}, \\
M_{22}=\left(1-\left|p_{23}\right|^{2}\right)^{-1 / 2}, \\
M_{23}=-p_{23}^{*}\left(1-\left|p_{23}\right|^{2}\right)^{-1 / 2}, \\
M_{33}=1,
\end{gathered}
$$

where

$$
\begin{gathered}
N=\left(1-\left|p_{23}\right|^{2}\right)^{-1}\left(1-\left|p_{23}\right|^{2}-\left|p_{12}\right|^{2}-\left|p_{13}\right|^{2}\right. \\
\left.+p_{12} p_{23} p_{13}^{*}+p_{12}^{*} p_{23}^{*} p_{13}\right), \\
p_{i j}=p_{j i}^{*}=\left\langle\theta_{\omega, i}^{\mathrm{st}-\mathrm{p}} \mid \theta_{\omega, j}^{\mathrm{st}-\mathrm{p}}\right\rangle_{\mathcal{W}-K G} .
\end{gathered}
$$

It is easy to check that the new modes are indeed orthonormal. Then the corresponding annihilation operators transform with the transposed inverse of the matrix $M$ :

$$
\left(\begin{array}{c}
a_{\omega, 1}^{\mathrm{st}} \\
a_{\omega, 2}^{\mathrm{st}} \\
a_{\omega, 3}^{\mathrm{st}}
\end{array}\right)=\left(M^{-1}\right)^{T}\left(\begin{array}{c}
a_{\omega \omega,}^{\mathrm{st}} \\
a_{\omega \omega, 2}^{\mathrm{st}} \\
a_{\omega, 3}^{\mathrm{st}-\mathrm{p}}
\end{array}\right) .
$$

Notice that $M$ is an upper triangular matrix. Therefore, the matrix $\left(M^{-1}\right)^{T}$ appearing in this equation is lower triangular. Also, $M^{T}$, which allows us to write the annihilation operators $a_{\omega, i}^{\mathrm{st}-\mathrm{p}}$ as linear combinations of $a_{\omega, i}^{\mathrm{st}}$, is a lower triangular matrix. This implies that $a_{\omega, 1}^{\mathrm{st}-\mathrm{p}}, a_{\omega, 2}^{\mathrm{st}-\mathrm{p}}$ depend linearly on $a_{\omega, 1}^{\mathrm{st}}, a_{\omega, 2}^{\mathrm{st}}$ only, and vice versa, never mixing with the third mode, which would lead to particle presence in the right asymptotic region.

Consider the stationary vacuum state defined by the requirement

$$
a_{\omega, i}^{\mathrm{st}}\left|0_{\mathrm{st}}\right\rangle=0 .
$$

The important point is that this state does not contain any quasiparticle coming in from or out at the right asymptotic region. To realize that this is indeed so, one has to check that the Bogolubov $\beta$ coefficients defined by the products $\left\langle\theta_{\omega, i}^{\text {st }} \mid \theta_{\omega, 1}^{\text {out } *}\right\rangle_{\mathcal{W} \text {-KG }}$ and $\left\langle\theta_{\omega, i}^{\text {st }} \mid \theta_{\omega, 2}^{\text {in } *}\right\rangle_{\mathcal{W} \text {-KG }}$ are identically zero $(i=1,2,3)$. Given the relation between the $\theta_{\omega, i}^{\text {st }}$ and the $\theta_{\omega, i}^{\mathrm{st}-\mathrm{p}}$ modes, this follows straightforwardly from the following argument.

By the definition of $\theta_{\omega, 1}^{\mathrm{st}-\mathrm{p}}$ and $\theta_{\omega, 2}^{\mathrm{st}-\mathrm{p}}$ it is obvious that $\left\langle\theta_{\omega, i}^{\mathrm{st}-\mathrm{p}} \mid \theta_{\omega, 1}^{\text {out* }}\right\rangle_{\mathcal{W} \text {-KG }}=0$ and $\left\langle\theta_{\omega, i}^{\mathrm{st}-\mathrm{p}} \mid \theta_{\omega, 2}^{\text {in* }}\right\rangle_{\mathcal{W} \text {-KG }}=0$ for $i=$ 1 , 2. In order to show that $\left\langle\theta_{\omega, 3}^{\mathrm{st}} \mid \theta_{\omega, 2}^{\text {in* }}\right\rangle_{\mathcal{W} \text {-KG }}=0$, evaluate the spatial integral that defines this inner product at $t \rightarrow$ $-\infty$. Then, it can be seen from the scattering diagrams that the involved modes do not have intersecting support, and therefore their product vanishes. A similar argument applies for $\left\langle\theta_{\omega, 3}^{\mathrm{st}-\mathrm{p}} \mid \theta_{\omega, 1}^{\text {out } *}\right\rangle_{\mathcal{W} \text {-KG }}$ by evaluating it at $t=+\infty$. (Note that this can be seen even more clearly by using a wave packet basis.)
Therefore in the stationary vacuum state there is no quasiparticle coming in from or out at the right asymptotic region. In other words, this state is a generalization to this dispersive theory of the Boulware stationary state for black holes and static stars.

In standard (nondispersive) general relativity, the Boulware state is not regular at the horizon. Indeed, the modes that characterize this state are not well defined there, because of the infinite blueshift associated with the horizon. In a Bose-Einstein condensate, the modes that mimic Boulware modes (i.e., those that characterize the stationary vacuum introduced above) are perfectly well defined across the horizon because Lorentz symmetry breaking in a Bose-Einstein condensate prevents the presence of infinite blueshifts at the (now nonstrict) horizon. Consequently, it should, in principle, be an attainable state in such a dispersive theory. In other words, it should, in principle, be possible to design a black hole configuration in a Bose-Einstein condensate not producing Hawking radiation.

\section{State preparation}

Given a stationary configuration, one could prepare the system to be in any of the vacuum states described above. The in vacuum contains quasiparticles coming out at the right infinity $(x \rightarrow+\infty)$, while the stationary vacuum state does not (it does not contain quasiparticles coming in from the right infinity either). In standard general relativity, one can prove that starting from a Minkowskian spacetime at past infinity and a relativistic field initially in its Minkowski vacuum, if one dynamically produces a black hole horizon, then the vacuum state at future infinity is indistinguishable from the Unruh state [21,22]. In particular, if one takes a Lorentz invariant theory and engineers, by using external means, a quasistatic collapse towards the formation of a black hole, then before the formation of the horizon, one can always make the collapse slow enough so that an initial Minkowskian state first acquires and then maintains, at each instant of time, the Boulware vacuum structure, which is the appropriate vacuum state for a stationary star. However, no matter how slow the collapse is, the quasistatic approximation breaks down when the horizon forms due to the infinite slow-down of clock rates at the horizon. As a consequence, the Boulware state is unavoidably modified and becomes, after some transient phase, the Unruh state with its associated Hawking emission. Note that this is also necessary for consistency: The renormalized stress-energy tensor in the Boulware state is divergent at the horizon and the state itself is not well defined there, because of the infinite blueshift of the corresponding modes caused by the presence of the (nondispersive) horizon, as pointed out above. The Unruh state, on the contrary, is perfectly regular at the (future) horizon (we do not mention here states, such as the HartleHawking state, which are not vacuum states in the past). 
Thus, within standard general relativity a field in a black hole spacetime can only be in the Unruh vacuum state (obviously, modulus the presence of any finite amount of particles) and never in the Boulware vacuum state.

In dispersive theories, the previous arguments are no longer valid and, depending on the specific preparation of the configuration-for instance, the specific dynamical way in which one sets up the subsonic-to-supersonic transition in the Bose-Einstein condensate flow-one could, in principle, end up having different final states for the final stationary flow. Indeed, in a Bose-Einstein condensate there is no infinite delay of the clocks, and so it should be possible to maintain the Boulware vacuum structure even after the horizon has been formed. In principle, one could, for example, end up in the stationary vacuum state described above, which is a regular extrapolation of the Boulware vacuum to the dispersive theory. Therefore, although, in general, the formation of a horizon in a Bose-Einstein condensate leads to a Hawking-like radiation associated with the in vacuum (as shown numerically in [10] and studied in detail in [17]), it remains to be seen which the precise sufficient conditions are in order to recover the presence of such Hawking radiation in systems with superluminal dispersion relations. This could, for instance, be relevant when analyzing the effect of highfrequency superluminal dispersion in proper black hole configurations and the influence of the black hole's internal region.

This issue has recently created some controversy. In Ref. [18] the present authors argued that, under the assumption that a quasistatic condition for the creation of a black hole applies, one does not need to take into account all the modes of the system to calculate the quasiparticle content at the right infinity, but only those that can be traced as rays escaping from the black hole configuration just before the actual horizon was formed. Quasiparticle production due to the formation of a black hole in the lab would then appear as just a transient regime that disappears in time: The system settles down to the stationary state described above. However, other authors claim that the relevant state for the final configuration is the in vacuum state (see, for example, [20]). This vacuum state produces an everlasting stationary stream of quasiparticles traveling towards the right asymptotic region, mimicking, in this way, a Hawking flux (the precise spectrum acquires some deviations with respect to a perfect blackbody but, in normal situations, these deviations are relegated to the high-frequency tail of the spectrum). If the in vacuum state were the only one available for the configuration, one could conclude that it is not possible to create a stable sub-tosupersonic transition in the lab: One would have to maintain it there by external means or it would eventually dissolve due to backreaction. This is what happens in standard general relativity: The existence of stationary black holes is not semiclassically consistent; they have to evaporate.
In the Bose-Einstein-condensate dispersive theory, the availability of the stationary vacuum implies that, in principle, it should be possible to produce a semiclassically stable analogue of a stationary black hole in the lab. Which is the precise vacuum state selected by the dynamical formation of the black hole horizon configuration under discussion remains to be calculated.

\section{CONCLUSIONS AND COMMENTS}

When establishing a gravitational analogy in condensedmatter systems, a low-energy Klein-Gordon dynamics emerges for the perturbations around a background configuration. In this paper, we have addressed the issue of the appropriate formulation for dealing with the quantum dynamics of such fluctuations: the one obtained by directly dealing with the quantum creation and annihilation operators for the excited quasiparticles (the Bogolubov formalism), or the relativistic approach consisting in obtaining a generalized Klein-Gordon equation and dealing with it in the relativistic quantum-field-theoretic way. This question applies, in particular, to the quasiparticle Hawking radiation when the background configuration supports an acoustic black hole, either stationary or externally generated. As we have seen, both methods are entirely equivalent, leading to the same quasiparticle concept, and hence to the same description.

It should be stressed that these results are actually more general than presented here. Indeed, if instead of a BoseEinstein condensate we had considered an arbitrary barotropic, inviscid and irrotational fluid described by an arbitrary enthalpy function $h(n)$, we would have drawn the same conclusions. Furthermore, for regimes in which the quantum potential is not significant (long wavelengths), the fluid is described in pure hydrodynamical terms and a proper Klein-Gordon equation is recovered. Therefore, we can conclude that the relativistic analogy with a hydrodynamic fluid is actually more than just an analogy: There is a complete equivalence leading to a hydrodynamical description of relativistic massless scalar fields and vice versa, even at the quantum level and in strong gravity regimes such as black holes.

Once the inner product is defined, one can find a complete and orthonormal set of positive norm modes, and an associated set of negative norm ones. One can then define quasiparticle creation and annihilation operators and make a choice of vacuum state. This procedure, as is well known, can be carried out in many equally valid ways, related through Bogolubov transformations. To illustrate the procedure, we considered a simple configuration of special interest with regard to the possible experimental detection of analogue Hawking radiation: a one-dimensional configuration simulating the presence of a black hole horizon for acoustic perturbations or, in other words, a flow with a subsonic-to-supersonic transition. 
In the last section, we have shown that there are several sets of modes and vacuum states with a particularly simple interpretation: the in modes and the out modes (with their associated in vacuum and out vacuum states), already present in previous analyses in the literature [20], and the stationary modes and stationary vacuum state, presented here for the first time. Contrarily to standard general relativity, the dispersive character of the Bogolubov theory allows the existence of a regular vacuum state which does not contain any incoming or outgoing quasiparticles in the external asymptotic region, namely, the stationary state. This state is a regular generalization of the notion of the Boulware state in black hole physics. Since it is regular, this state can, in principle, be attained by adequately preparing the system. In general, the formation of a horizon in a Bose-Einstein condensate should lead to a Hawkinglike radiation associated with the in vacuum [10,17]. However, in the light of the present discussion, it should at least theoretically be possible to produce a black hole analogue in a Bose-Einstein condensate without causing the emission of a stationary Hawking flux. Whether a black hole analogue in a Bose-Einstein condensate radiates or not might depend on the specific path followed in setting up the configuration. This could be an important issue for experimentalists trying to reproduce Hawking radiation in a lab. From a relativistic point of view, it could also be of considerable importance when analyzing the effect of modified dispersion at high energies in gravitational black hole configurations in which the characteristics of the internal region are uncertain. We will return to these issues in future studies.

\section{ACKNOWLEDGMENTS}

Financial support was provided by the Spanish MICINN through Projects No. FIS2008-06078-C03-01 and No. FIS2008-06078-C03-03 and Consolider-Ingenio 2010 Program CPAN (CSD2007-00042) and by the Junta de Andalucía through Projects No. FQM2288 and No. FQM219. The authors want to thank S. Finazzi, S. Liberati, G. A. Mena Marugán, and R. Parentani for illuminating discussions.
[1] R. W. White, J. Acoust. Soc. Am. 53, 1700 (1973).

[2] W. G. Unruh, Phys. Rev. Lett. 46, 1351 (1981).

[3] M. Visser, Classical Quantum Gravity 15, 1767 (1998).

[4] C. Barceló, S. Liberati, and M. Visser, Living Rev. Relativity 8, 12 (2005), http://relativity.livingreviews.org/ Articles/lrr-2005-12.

[5] G. E. Volovik, Ann. Phys. (N.Y.) 14, 165 (2005).

[6] C. Barceló, L. J. Garay, and G. Jannes, arXiv:1002.4651.

[7] L. J. Garay, J. R. Anglin, J. I. Cirac, and P. Zoller, Phys. Rev. Lett. 85, 4643 (2000).

[8] L. J. Garay, J. R. Anglin, J. I. Cirac, and P. Zoller, Phys. Rev. A 63, 023611 (2001).

[9] O. Lahav, A. Itah, A. Blumkin, C. Gordon, and J. Steinhauer, arXiv:0906.1337.

[10] I. Carusotto, S. Fagnocchi, A. Recati, R. Balbinot, and A. Fabbri, New J. Phys. 10, 103001 (2008).

[11] S. Liberati, M. Visser, and S. Weinfurtner, Classical Quantum Gravity 23, 3129 (2006).

[12] C. Barceló, A. Cano, L. J. Garay, and G. Jannes, Phys. Rev. D 75, 084024 (2007).
[13] Y. Kurita, M. Kobayashi, T. Morinari, M. Tsubota, and H. Ishihara, Phys. Rev. A 79, 043616 (2009).

[14] A. L. Fetter, Ann. Phys. (N.Y.) 70, 67 (1972).

[15] Y. Castin, arXiv:cond-mat/0105058.

[16] S. Corley and T. Jacobson, Phys. Rev. D 59, 124011 (1999).

[17] J. Macher and R. Parentani, Phys. Rev. A 80, 043601 (2009).

[18] C. Barceló, L. J. Garay, and G. Jannes, Phys. Rev. D 79, 024016 (2009).

[19] C. Barceló, S. Liberati, S. Sonego, and M. Visser, New J. Phys. 6, 186 (2004).

[20] J. Macher and R. Parentani, Phys. Rev. D 79, 124008 (2009).

[21] R. M. Wald, Quantum Field Theory in Curved Space-Time and Black Hole Thermodynamics (Chicago University Press, Chicago, 1994).

[22] I. Racz and R. M. Wald, Classical Quantum Gravity 9, 2643 (1992). 